Original Paper

http://indexmedicus.afro.who.int

\title{
Caractérisation agromorphologique des sorghos à grains sucrés (Sorghum bicolor (L.) Moench) du Burkina Faso
}

\author{
Nerbéwendé SAWADOGO $^{1 *}$, Baloua NEBIE ${ }^{1,2}$, Mariam KIEBRE ${ }^{1}$, \\ Pauline Bationo KANDO ${ }^{1}$, Romaric Kiswendsida NANEMA ${ }^{1}$, \\ Renan Ernest TRAORE ${ }^{1}$, Gapili NAOURA ${ }^{1,3}$, Mahamadou SAWADOGO ${ }^{1}$, \\ Jean-Didier ZONGO ${ }^{1}$ \\ ${ }^{1}$ Université de Ouagadougou, Laboratoire Biosciences, Equipe Génétique et Amélioration des plantes, \\ 03 BP 7021 Ouagadougou 03, Burkina Faso. \\ ${ }^{2}$ International Crops Research Institute for the Semi-Arid Tropics (ICRISAT). \\ ${ }^{3}$ Institut Tchadien de Recherche Agronomique pour le Développement (ITRAD), Tchad. \\ *Auteur correspondant, E-mail: nerbewende@yahoo.fr ; alifekhaled2010@gmail.com, \\ Tél : (+226) $76578949 /(+226) 70384694$
}

\section{RESUME}

Au Burkina Faso, le sorgho à grains sucrés [Sorghum bicolor (L.) Moench] est une culture marginale dont les grains sont consommés frais au stade pâteux. Afin de recueillir les connaissances paysannes sur la gestion du sorgho à grains sucrés et de déterminer le niveau et la structuration de la diversité agromorphologique de ces sorghos du Burkina, une prospection-collecte d'accessions a été réalisée dans les quatre zones agro-écologiques du pays. Au total, 126 accessions ont été collectées dans 102 villages parmi lesquelles 97 ont été caractérisées à partir de 12 caractères qualitatifs et 12 quantitatifs suivant un dispositif blocs de Fisher à trois répétitions. L'étude a montré d'une part que ce sorgho est cultivé surtout dans les champs de case et principalement par l'ethnie mossi et d'autre part que la nomenclature paysanne est basée surtout sur les caractères phénotypiques visibles. Une importante variabilité agromorphologique a été observée au niveau de tous les caractères quantitatifs et une répartition des 97 accessions en 3 groupes : les accessions à cycle long et à rendement grains élevé, les accessions à cycle court et à rendement grains faible et les accessions à cycle et rendement moyens. Les races botaniques caudatum $(45,4 \%)$ et caudatum-guinea $(48,5 \%)$ sont les plus prédominantes dans la collection. Cette diversité génétique est un atout important pour les programmes de sélection et d'amélioration génétique du sorgho.

(C) 2014 International Formulae Group. All rights reserved.

Mots clés : Prospection-collecte, variabilité agromorphologique, Burkina Faso.

\section{INTRODUCTION}

Le sorgho (Sorghum bicolor L. Moench) est la principale culture céréalière au Burkina Faso. Sa production est estimée à 1,7 millions de tonnes, représentant $44 \%$ de la production céréalière totale (Faostat, 2012).
En plus du sorgho non sucré cultivé pour son grain utilisé dans la préparation de plusieurs mets, d'autres types de sorgho dont le sorgho à grains sucrés sont rencontrés dans plusieurs zones agroécologiques. 
Le sorgho à grains sucrés est principalement cultivé pour ses grains qui sont consommés à l'état pâteux. Il est généralement récolté avant les principales cultures vivrières et constitue de ce fait un aliment de soudure en zone rurale (Nebié et al., 2012). De plus, les feuilles et les tiges sont exploitées comme fourrage ou comme combustible. Comparativement aux principales cultures céréalières (sorgho non sucré, mil, maïs), le sorgho à grains sucrés est une plante mineure dans le système agraire burkinabè. Sa culture est de plus en plus abandonnée menaçant ainsi la préservation de ses ressources génétiques. Malgré ces difficultés, les variétés traditionnelles demeurent une source importante de gènes dont la sauvegarde contribue au maintien de la richesse génétique (Frankel et al., 1995).

Les études de diversité génétique des sorghos du Burkina Faso se sont plus intéressées aux sorghos non sucrés (Zongo, 1991 ; Barro/kondombo, 2010) et aux sorghos à tige sucrée (Nebié, 2014). Elles ont révélé une importante variabilité au niveau des marqueurs morphologiques et moléculaires. La diversité génétique des sorghos à grains sucrés du Burkina Faso reste mal connue. Seule une étude d'ordre morphologique d'écotypes du Centre nord du pays (Nebié et al., 2012) a été effectuée. Elle a montré une prédominance des races bicolor et caudatumguinea.

L'objectif de cette étude est de recueillir les connaissances paysannes sur la gestion du sorgho à grains sucrés du Burkina Faso d'une part, et d'autre part de déterminer le niveau et la structuration de la diversité agromorphologique de ces sorghos.

\section{MATERIEL ET METHODES Prospection-collecte}

La prospection-collecte a été effectuée entre 2008 et 2010 dans 102 villages repartis dans les quatre zones agro-écologiques du Burkina Faso (Figure 1). L'identification des villages où le sorgho à grains sucrés est cultivé et la collecte des accessions ont été faites avec l'aide des agents des services déconcentrés du Ministère de l'Agriculture et de la Sécurité alimentaire. Dans la mesure où la culture de ce sorgho est faite à petite échelle, la technique de collecte exhaustive a été utilisée en enquêtant tous les producteurs de chaque village prospecté. Chez chaque producteur, les panicules de tous les morphotypes de sorgho à grains sucrés cultivés ont été collectées, soit 126 accessions provenant de 102 villages repartis dans 14 provinces.

La collecte des accessions auprès de chaque producteur a été suivie d'une interview semi-structurée. Ces interviews ont permis de recueillir les informations sur l'origine de l'accession, l'appellation locale, les méthodes de conservation des semences, les pratiques culturales ainsi que les usages.

\section{Caractérisation agro-morphologique des accessions collectées \\ Matériel végétal \\ Un total de 126 accessions a été} collecté sur les différents sites de culture du sorgho à grains sucrés au Burkina Faso, parmi lesquelles, 97 accessions ont été retenues pour l'évaluation agromorphologique à cause de la mauvaise qualité germinative des autres accessions.

\section{Site et dispositif expérimental}

Les essais ont été conduits en juillet 2012 dans la station expérimentale de Gampèla située à $1^{\circ} 21^{\prime}$ de longitude Ouest et $12^{\circ} 24$ 'de latitude Nord. De juillet à octobre 2012, une pluviométrie cumulée de $848,5 \mathrm{~mm}$ a été enregistrée en 35 jours. Le dispositif expérimental est un bloc de Fisher à trois répétitions. Les répétitions ont été séparées par une allée de $2 \mathrm{~m}$. Dans une répétition, chaque accession est semée sur une ligne de 5,6 $\mathrm{m}$ avec un écartement de $0,8 \mathrm{~m}$ entre les lignes et $0,4 \mathrm{~m}$ entre les poquets. De l'engrais NPK à la dose de $100 \mathrm{~kg} / \mathrm{ha}$ et de l'urée (50 $\mathrm{kg} / \mathrm{ha}$ ) ont été respectivement apportés au labour et au stade gonflement de la panicule.

\section{Caractères étudiés}

24 caractères dont 12 qualitatifs et 12 quantitatifs choisis pour la plus part dans le descripteur du sorgho (IBPGR/ICRISAT, 1993) ont été utilisés pour la caractérisation des accessions. 
Les caractères qualitatifs ont concerné la couleur de la plantule à la levée (CPL); l'inflorescence notamment la forme du pédoncule (FPE), l'exsertion (EXE) et le type de panicule (TPA); le grain et l'épillet à savoir la forme (FGR), la couleur (CGR), la vitrosité (VIT), la couverture du grain par les glumes (COG), la rotation (ROT), la couleur des glumes (CGL) et la présence de l'aristation (ARI).

Les caractères type de panicule, couverture du grain par les glumes, forme et rotation du grain ont servi à la détermination des types botaniques (TBO) grâce à la clé de Harlan et De Wet (1972).

Les caractères quantitatifs mesurés sont la hauteur de la plante (HPL), le cycle $50 \%$ floraison (NJF), le diamètre de la tige (DTI), la longueur (LOE) et le nombre (NEN) d'entrenœuds, la longueur (LOF) et la largeur (LAF) de la troisième feuille sous paniculaire, la longueur (LOP), la largeur (LAP) et le poids de la panicule principale (PPA), le poids de cent grains (PCG) et le rendement grains par plante (GPL).

A l'exception du nombre de jours, $50 \%$ floraison évalué sur toute la ligne et du poids de cent grains déterminé au stade grains secs, tous les autres ont été mesurés au stade grains pâteux sur trois plants aléatoirement choisis par ligne.

\section{Analyses statistiques}

Les logiciels Excel, Genstat, v4.10.3 (VSN International Ltd, 2011) et Xlstat pro version 7.1 (htt://www .XLSTAT.com/fr) ont été utilisés pour analyser les données collectées. Excel a servi au calcul des fréquences des résultats de l'enquête et des caractères qualitatifs et Genstat v4.10.3 aux analyses de variance. Pour l'ensemble des caractères quantitatifs étudiés, l'héritabilité au sens large $\left(\mathrm{H}^{2}\right)$ a été calculée a partir des variances génotypique (VG) et phénotypique (VP), données par Genstat, selon la formule de Rex (2002): $H^{2}(\%)=(V G / V P) * 100$. Les relations entre les caractères, la structuration de la variabilité et la caractérisation des groupes ont été effectuées avec Xlstat pro.7.1. La classification ascendante hiérarchique a été réalisée selon la méthode d'agrégation de Ward à partir des moyennes de six caractères quantitatifs ayant trait à la croissance végétative (LAF, LOE, HPL), au rendement (GPL), au cycle (NJF) et à la panicule (LAP).

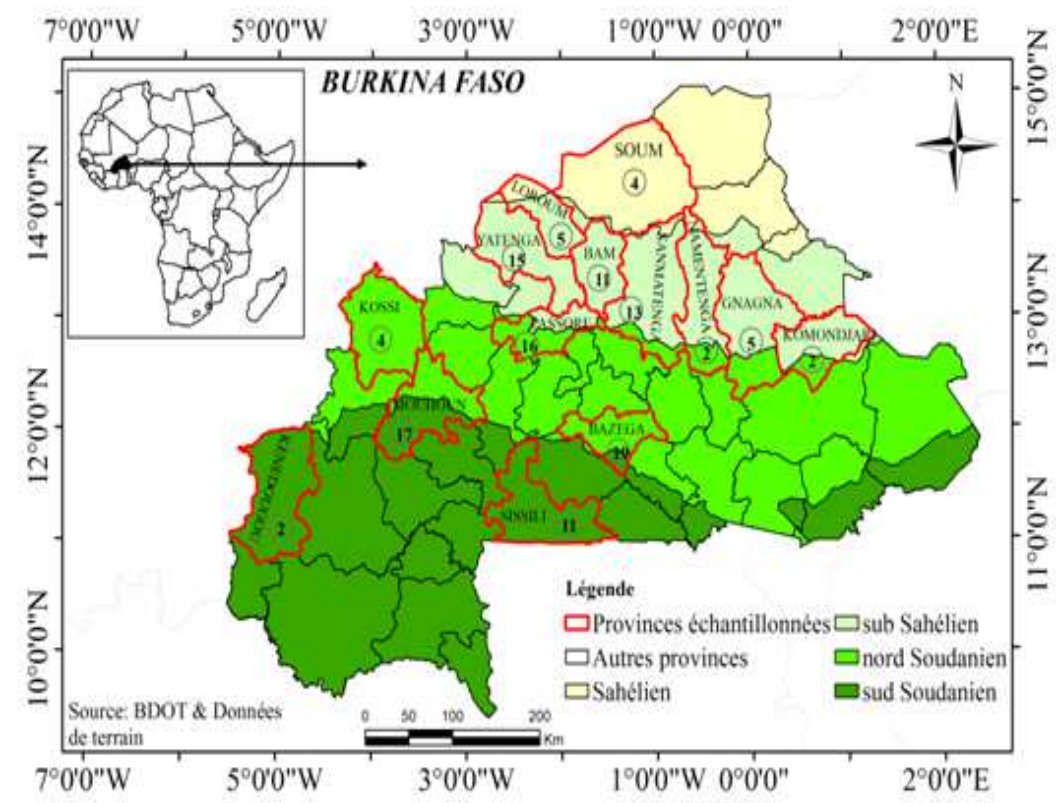

Figure 1 : Répartition des accessions en fonction de leurs origines géographiques. 


\section{RESULTATS}

\section{Description de la collection de sorghos à grains sucrés}

Les résultats de l'enquête (Tableau 1) montrent que la culture du sorgho à grains sucrés est pratiquée par plusieurs groupes ethniques mais le groupe le plus rencontré est celui des mossis $(89,7 \%)$. Les appellations varient entre les ethnies et à l'intérieur de la même ethnie selon les localités surtout chez les mossis qui leur attribuent plus d'une quinzaine de noms. Ces différents groupes ethniques désignent les accessions par des noms en rapport avec leur origine ou des caractères phénotypiques comme la saveur du grain, la forme de la panicule, le mode d'égrenage ou la couleur du grain. Ainsi on rencontre les appellations comme maka (Mossi) pour désigner les accessions qui seraient originaires de la Mecque, wombednoogo (Mossi), dimangar (Goumantché), dimemegou (Goumantché) et raptirga (Peulh) en référence à la saveur sucrée du grain, walvèguelobé (Mossi) pour désigner la forme en nids de tourterelle de la panicule, kassaarga ou zoussaarga (Mossi), samadjaka (Peulh) en relation avec la facilité d'égrenage et Bimi-wili (Dafi) en rapport à la couleur rouge des grains. La culture de ce sorgho est essentiellement réalisée dans le terroir mossi (Bazèga, Bam, Namentenga, Lorum, Passoré, Yatenga).

L'analyse des modes de gestion consignée dans le Tableau 2 montre que les paysans pratiquent la monoculture et l'association culturale avec une prédominance marquée de la culture associée (94,3\%). Le sorgho à grains sucrés est principalement cultivé dans les champs de case $(88,7 \%)$ et associé à des cultures comme le niébé, le maïs et les sorghos non sucrés. Les panicules sont conservées comme semences dans des enceintes hermétiques telles que les sachets plastiques, les bouteilles, les bidons $(66,7 \%)$ ou simplement accrochées aux arbres, aux hangars ou aux maisons (32,3\%). Il est un sorgho de bouche récolté à l'état pâteux pour la consommation familiale et/ou la vente.

\section{Variation des caractères qualitatifs}

Les résultats de l'analyse des caractères qualitatifs du Tableau 3 ont montré que toutes les accessions sont anthocyanées et présentent un pédoncule dressé, une exsertion positive, des grains farineux. Les autres caractères ont révélé une grande variabilité. Ainsi, les sorghos à grains sucrés présentent des plantules de couleur verte $(87,6 \%)$ ou violette $(12,4 \%)$, des glumes de couleur marron $(46,4 \%)$, paille $(38,1 \%)$ ou noire $(15,5 \%)$, des grains de couleur rouge $(90,7 \%)$ ou grise $(9,3 \%)$ majoritairement non aristés $(87,6 \%)$. Les grains sont de forme dissymétrique $(90,7 \%)$ ou elliptique $(9,3 \%)$ et couverts pour la plus part sur au plus 50\% (90,7\%) avec une rotation faible $(40,2 \%)$ ou absente $(46,4 \%)$. Trois types de panicules sont observés (lâche, très lâche, semi-compacte) avec une prédominance de la forme lâche $(78,4 \%)$. En se basant sur la classification de Harlan et De Wet (1972) axée sur la forme du grain et les caractères de l'épillet, $45,4 \%$ des accessions sont des caudatum, $1 \%$ des bicolor et $53,6 \%$ sont des formes intermédiaires dont $48,5 \%$ de caudatum-guinea et $5,1 \%$ de guinea-bicolor.

\section{Variation des caractères quantitatifs}

Les résultats de l'analyse de variance présentés dans le Tableau 4 montrent qu'à l'exception du diamètre de la tige discriminant seulement à $5 \%$, tous les autres caractères discriminent les accessions au seuil de $5 \%$ et $1 \%$, ce qui témoigne d'une variabilité morphologique importante entre elles. Le cycle 50\% floraison a varié de 61 à 91 jours avec $62,9 \%$ des accessions qui ont un cycle inférieur à 80 jours. Les types botaniques caudatum et guinea-bicolor ont les cycles extrêmes respectifs de 80,7 jours et 69,1 jours. La largeur des feuilles a varié de 6 à $13 \mathrm{~cm}$, la longueur de 64,3 à $89,3 \mathrm{~cm}$, la hauteur de la plante de 199,7 à $398 \mathrm{~cm}$ pour un diamètre allant de 1,2 à $2,7 \mathrm{~cm}$ et un nombre moyen d'entrenœuds de 10,9. Les valeurs moyennes de la longueur de l'entrenœud et de la hauteur des plantes ont été respectivement de $23,3 \mathrm{~cm}$ et $285,3 \mathrm{~cm}$. Les panicules principales sont 
longues de $20,7 \mathrm{~cm}$ à $51,3 \mathrm{~cm}$ et larges de 6,7 à $23,3 \mathrm{~cm}$ avec un poids moyen de $165 \mathrm{~g}$. Le rendement grains moyen par plante est de $136,7 \mathrm{~g}$ avec un poids de cent grains (PCG) variant de 1,3 à $3,7 \mathrm{~g}$.

Les valeurs du coefficient de variation indiquent une forte variation $(\mathrm{CV}>30 \%)$ entre les accessions pour le rendement grains et le poids de la panicule principale. Selon Aljane et Ferchichi (2007), une valeur élevée du coefficient de variation traduit une forte hétérogénéité du matériel étudié. Ainsi on observe une forte hétérogénéité entre les accessions pour le rendement grains et le poids de la panicule principale. L'héritabilité au sens large $\left(\mathrm{H}^{2}\right)$ varie de $83,2 \%$ pour le diamètre de la tige à $95,6 \%$ pour le cycle $50 \%$ floraison. Selon Stansfield (1983), l'héritabilité est élevée lorsque sa valeur est supérieure à $50 \%$. L'héritabilité au sens large est donc élevée pour tous les caractères.

Influence des facteurs « accessions », « race botanique » et « origine climatique» sur l'expression des caractères quantitatifs

Les coefficients de détermination $\left(\mathrm{R}^{2}\right)$ du Tableau 5 montrent que le facteur « origine climatique » a les plus faibles valeurs. La plus grande partie de cette variabilité est surtout expliquée par le facteur «accession» $\left(\mathrm{R}^{2}>55 \%\right.$ pour tous les caractères) suivi du facteur «race botanique». L'analyse de variance selon les facteurs accession et race botanique a montré que tous les caractères sont discriminants au seuil de $5 \%$ alors que selon l'origine climatique des accessions, seuls les caractères cycle $50 \%$ floraison, largeur de la feuille, nombre et longueur des entrenœuds, diamètre de la tige et poids de la panicule principale discriminent les accessions.

\section{Relations entre les caractères}

La matrice de corrélation de Pearson du Tableau 6 fait ressortir de nombreuses corrélations significatives aux seuils de $5 \%$ et $1 \%$. Ainsi le cycle $50 \%$ floraison est négativement et significativement corrélé au poids de cent grains $(r=-0,60)$ et la longueur de la panicule $(r=-0,24)$. Il est par contre positivement et très significativement corrélé au poids de la panicule principale $(\mathrm{r}=0.31)$ et au rendement grains par plante $(r=0,25)$. La hauteur de la plante est positivement et très significativement corrélée au nombre d'entrenœuds $(r=0,41)$ et à la longueur de l'entrenœud $(r=0,52)$. Le poids de cent grains est négativement et très significativement corrélé au poids de la panicule principale $(\mathrm{r}=$ $-0,33)$ et au rendement grains par plante $(r=$ $-0,28)$.

\section{Structuration de la variabilité des sorghos à grains sucrés}

Le dendrogramme de la classification ascendante hiérarchique de la Figure 2 révèle une répartition des 97 accessions en 3 groupes. La structuration obtenue est faiblement liée à l'origine climatique des accessions. Le test du Lambda de Wilks en analyse factorielle discriminante qui donne des valeurs de $F$ observé et $F$ critique respectives de 31,576 et 1,811 avec un p-value $<0,0001$ au seuil de 5\% entre les 3 groupes obtenus, montre qu'ils sont bien distincts.

L'analyse factorielle discriminante (AFD) des trois groupes de la Figure 3 montre que les deux premiers axes expliquent la totalité de la variabilité. Le premier axe qui l'explique à $91,06 \%$ oppose le cycle $50 \%$ floraison, la largeur de la feuille et le rendement grains à la longueur de l'entrenœud alors que l'axe 2, qui l'explique avec $8,94 \%$ oppose la largeur de la panicule à la hauteur de la plante. Les axes 1 et 2 sont respectivement ceux du rendement et de la taille. La relation des groupes avec les axes montre que les groupes I et III fortement corrélés à l'axe 1 sont opposés. Le groupe II par contre est très faiblement corrélé d'une part négativement à l'axe 1 et d'autre part positivement à l'axe 2 .

L'analyse de variance (Tableau 7) indique également que les groupes diffèrent significativement au seuil de $5 \%$ et $1 \%$ pour tous les caractères à l'exception de la longueur 
de la feuille discriminant seulement au seuil de 5\%. Le Tableau 7 résume les caractéristiques des groupes constitués.

Le groupe I est constitué de 35 accessions dont 32 caudatum et 3 caudatumguinea originaires de 10 provinces. Ce groupe se distingue par des accessions aux entrenœuds courts, feuilles larges, cycle long et rendement grains élevé. Le groupe II comprend 51 accessions originaires de 13 provinces reparties en 12 caudatum et 39 caudatum-guinea. Ce groupe se caractérise par des accessions aux panicules larges et aux valeurs moyennes pour les autres caractères. Quant au groupe III plus diversifié, il est constitué de 11 accessions dont 6 caudatumguinea, 1 bicolor et 5 guinea-bicolor issues de
7 provinces. Il se différencie des autres par des accessions aux entrenœuds longs, feuilles minces, cycle court et rendement grains faible. L'influence du facteur origine climatique dans la répartition des groupes est faible ( $\mathrm{F}$ value de 3,78 et une p-value de 0,013 au seuil de $5 \%$ ). En effet le groupe I est constitué majoritairement des accessions de la zone nord-soudanienne $(51,43 \%)$ alors que les groupes II et III renferment principalement les accessions de la zone sub-sahélienne à des proportions respectifs de $70,51 \%$ et $63,64 \%$. Les accessions du groupe II sont rencontrées dans toutes les zones agro-écologiques alors que les accessions des groupes I et III sont respectivement absentes dans les zones sahélienne et sud-soudanienne.

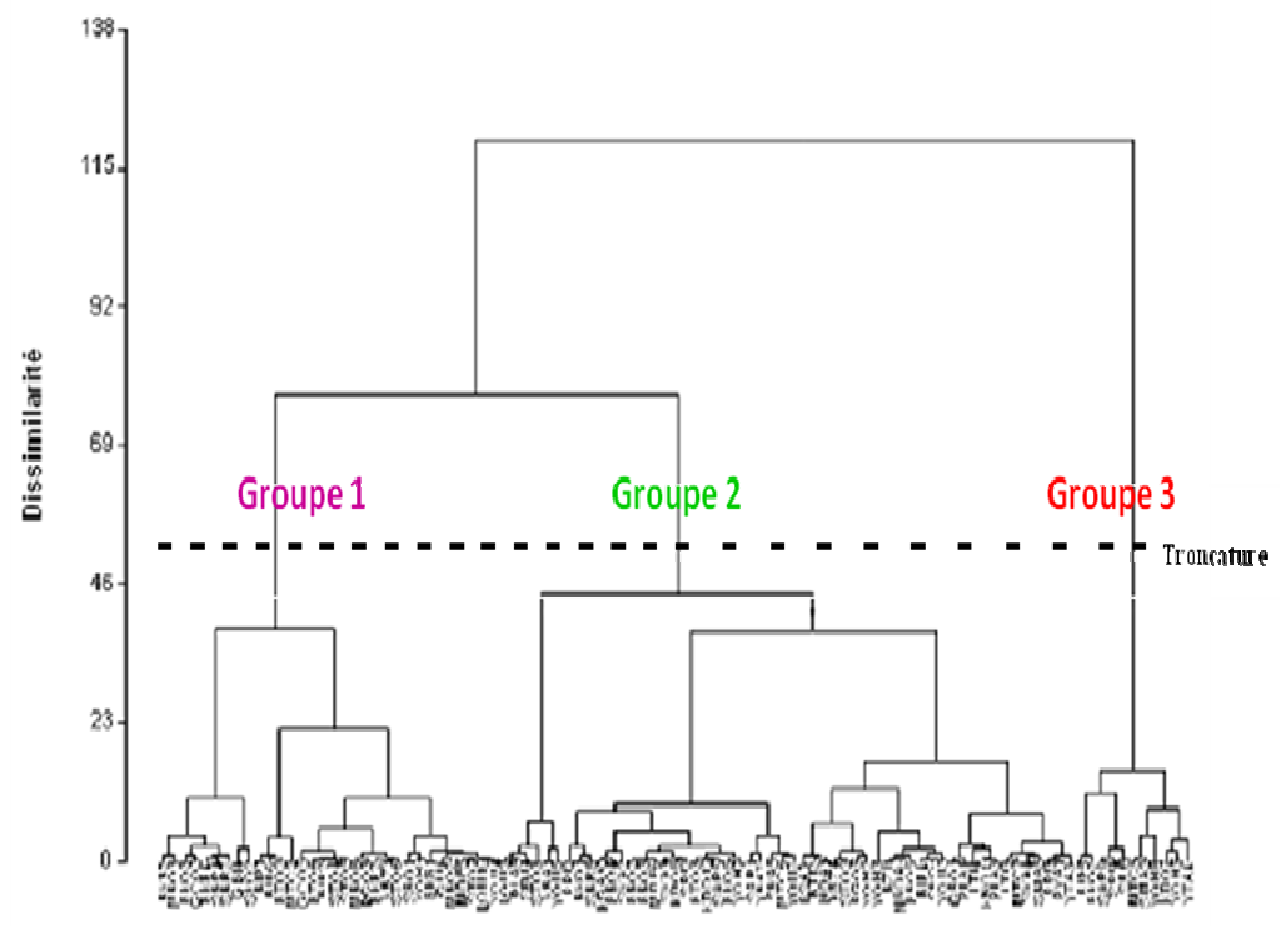

Figure 2 : Classification ascendante hiérarchique de la collection de sorghos à grains sucrés du Burkina Faso. 


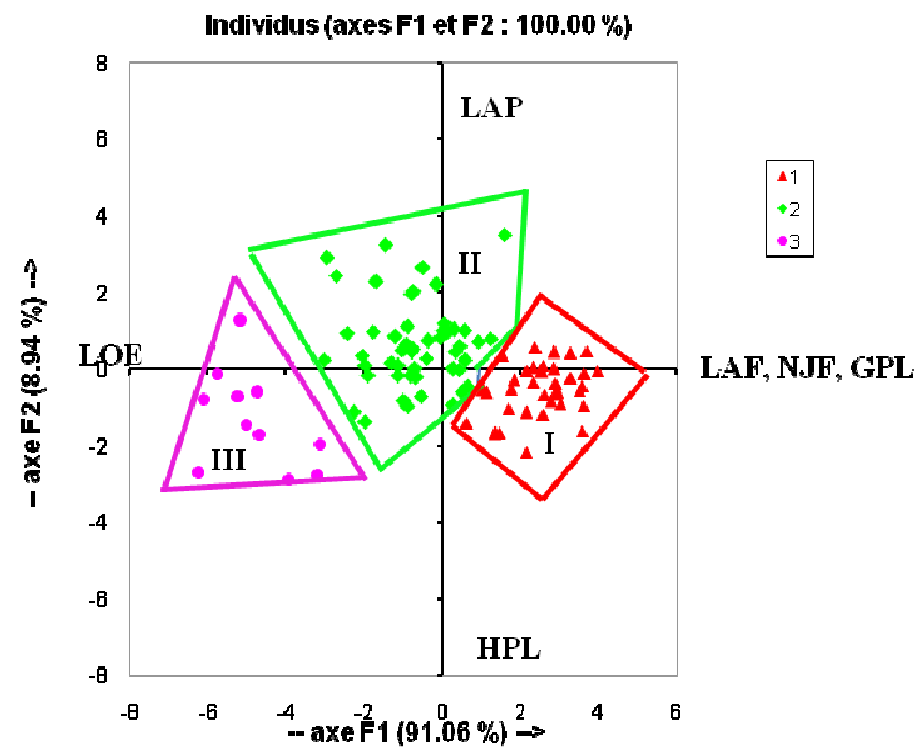

Figure 3: Position des groupes agromorphologiques de sorghos à grains sucrés en analyse factorielle.

Tableau 1 : Répartition des accessions de sorgho à grains sucrés par groupe ethnique et principales appellations locales.

\begin{tabular}{lccc}
\hline Groupe & Nombre & Proportion & Noms locaux \\
\hline Mossi & 113 & 89,7 & Maka, wombednoogo, walvèguelobé, \\
\hline Gourmantché & 06 & 4,8 & Didjié, dimangar, dimemegou \\
\hline Peulh & 03 & 2,3 & Raptirga, samadjaka \\
\hline Dafi & 03 & 2,4 & Yagnimi, bimi-wili, \\
\hline Bwabu & 01 & 0,8 & Sio \\
\hline
\end{tabular}

Tableau 2 : Modes de gestion paysanne des sorghos à grains sucrés au Burkina Faso.

\begin{tabular}{llc}
\hline Caractéristiques culturales & & Fréquence (\%) \\
\hline Mode de culture paysanne & Monoculture & 5,7 \\
& Association & 94,3 \\
\hline \multirow{3}{*}{ Type de champ } & Champ de case & 88,7 \\
& Champ de brousse & 6,5 \\
& Champ de case et de brousse & 4,8 \\
\hline Mode de conservation & Sachets plastiques, bidons ou bouteilles & 66,7 \\
des panicules & Accrochées aux arbres, aux maisons & \\
& ou aux hangars & 32,3 \\
\hline
\end{tabular}


Tableau 3 : Répartition des 97 accessions de sorghos à grains sucrés du Burkina Faso pour 9 caractères qualitatifs.

\begin{tabular}{|c|c|c|}
\hline Caractères & Modalités & Fréquence (en \%) \\
\hline & Vert & 87,6 \\
\hline \multirow[t]{3}{*}{ Couleur des plantules (CPL) } & Violacée & 12,4 \\
\hline & Gris & 9,3 \\
\hline & Rouge-clair & 58,7 \\
\hline \multirow[t]{4}{*}{ Couleur du grain (CGR) } & Rouge-foncé & 32 \\
\hline & Noir & 15,5 \\
\hline & Paille & 38,1 \\
\hline & Marron clair & 36,1 \\
\hline \multirow[t]{2}{*}{ Couleur des glumes (CGL) } & Marron foncé & 10,3 \\
\hline & Présence d'arête & 12,4 \\
\hline \multirow[t]{3}{*}{ Aristation (ARI) } & Absence d'arête & 87,6 \\
\hline & Couvert sur $1 / 4$ & 51,5 \\
\hline & Couvert sur $1 / 2$ & 39,2 \\
\hline \multirow[t]{2}{*}{ Couverture du grain (COG) } & Couvert sur $3 / 4$ & 9,3 \\
\hline & Dissymétrique & 90,7 \\
\hline \multirow[t]{3}{*}{ Forme du grain (FGR) } & Elliptique & 9,3 \\
\hline & Absent & 46,4 \\
\hline & Faible $\left(<90^{\circ}\right)$ & 40,2 \\
\hline \multirow[t]{3}{*}{ Rotation du grain (ROT) } & Fort $\left(90^{\circ}\right)$ & 13,4 \\
\hline & Très lâche & 14,4 \\
\hline & Lâche & 78,4 \\
\hline \multirow[t]{4}{*}{ Type de panicule (TPA) } & Semi-compacte & 7,2 \\
\hline & Caudatum & 45,4 \\
\hline & Caudatum-guinea & 48,5 \\
\hline & Guinea-bicolor & 5,1 \\
\hline Type botanique (BOT) & Bicolor & 1 \\
\hline
\end{tabular}

\section{DISCUSSION}

\section{Zones de culture et nomenclature paysanne}

Le sorgho à grains sucrés n'est pas très bien représenté sur l'ensemble du territoire du Burkina Faso. La culture est surtout pratiquée dans les zones subsahélienne et nord soudanienne par l'ethnie mossi. La faible représentativité de ce type de sorgho dans les autres zones serait liée aux conditions climatiques. La zone sud soudanienne plus arrosée (pluviométrie $\mathrm{P}>900 \mathrm{~mm}$ ) est propice à la culture du maïs et des tubercules (Traoré, 2014) et la zone sahélienne moins arrosée ( $\mathrm{P}<$ $500 \mathrm{~mm}$ ) à celle du mil (Nebié, 2014).

Les appellations paysannes tiennent compte des caractères phénotypiques du grain tels que la saveur, la couleur ou la forme de la panicule. Selon Djé et al. (2007), Deu et al. (2008), Missihoun et al. (2012) et Nebié (2014), la nomenclature des sorghos locaux est surtout basée sur des parties de la plante telles que le type de panicule, la couleur des grains, la couleur des glumes et la saveur de la tige. Des dénominations d'autres espèces selon des critères agromorphologiques avaient été également rapportées par plusieurs auteurs, à savoir Ouédraogo et al. (2010) sur le niébé, Nanema (2010) sur le fabirama, Abu et Buah (2011) sur le voandzou, Sawadogo et Balma (2003), et Jiro et al. (2011) sur le gombo. Ces dénominations paysannes s'expliqueraient par le mode de sélection paysanne basé essentiellement sur des caractères phénotypiques visibles. 
Tableau 4: Performances des accessions de sorghos à grains sucrés.

\begin{tabular}{lcccccc}
\hline Caractères & Min. & Max. & Moy. & CV $(\boldsymbol{\%})$ & $\mathbf{F}$ & $\mathbf{H}^{\mathbf{2}}(\boldsymbol{\%})$ \\
\hline NJF (jours) & 61 & 91 & 78,1 & 7,3 & $6,8^{* *}$ & 95,6 \\
LOF $(\mathrm{cm})$ & 64.3 & 89,3 & 75,4 & 6,4 & $2^{* *}$ & 89,4 \\
LAF $(\mathrm{cm})$ & 6 & 13 & 9,8 & 12,2 & $2,9^{* *}$ & 89,4 \\
NEN & 6,7 & 14,3 & 10,9 & 14,1 & $2,2^{* *}$ & 88,4 \\
LOE (cm) & 16,65 & 30,5 & 23,3 & 9,5 & $3,2^{* *}$ & 90,9 \\
HPL (cm) & 199,7 & 398 & 285,3 & 11 & $2,6^{* *}$ & 90,3 \\
DTI (cm) & 1,23 & 2,7 & 1,8 & 15,4 & $1,6^{*}$ & 83,2 \\
LOP (cm) & 20,7 & 51,3 & 33,4 & 14,9 & $4,7^{* *}$ & 93,7 \\
LAP (cm) & 6,7 & 23,3 & 13,8 & 21,2 & $4,8^{* *}$ & 93,9 \\
PPA (g) & 32,8 & 302,1 & 165 & 30,9 & $2,3 * *$ & 87,5 \\
GPL (g) & 16,6 & 333,5 & 136,7 & 42,1 & $2,2^{* *}$ & 86,8 \\
PCG (g) & 1,3 & 3,7 & 2,3 & 16,1 & $3,9^{* *}$ & 92,2 \\
\hline
\end{tabular}

NJF : nombre de jours à $50 \%$ floraison, LOF : longueur de la $3^{\text {ieme }}$ feuille sous paniculaire, LAF : largeur de la $3^{\text {ième }}$ feuille sous paniculaire, NEN : nombre d'entre-nœuds, LOE : longueur de l'entre-nœud, HPL : hauteur de la plante, DTI : diamètre de la tige principale, LOP : longueur de la panicule, LAP : largeur de la panicule, PPA : poids de la panicule principale à l'état pâteux, GPL : rendement en grains par plante à l'état pâteux, PCG : poids de cent grains, Min.: minimum, Max. : maximum, Moy.: moyenne; CV : coefficient de variation, F: coefficient de Fischer, *: différence significative à $5 \%$, ** : différence significative à $1 \%, \mathrm{H}^{2}$ : héritabilité au sens large.

Tableau 5: Comparaison des effets de l'origine climatique, de la race botanique et des accessions sur l'expression des caractères des sorghos à grains sucrés du Burkina Faso.

\begin{tabular}{|c|c|c|c|c|c|}
\hline Caractères & F (o. cli) & F (race) & $\mathbf{R}^{2}$ o. cli (\%) & $\mathbf{R}^{2}$ race $(\%)$ & $\mathbf{R}^{2}$ accession (\%) \\
\hline NJF (jours) & $32 *$ & $35,7 * *$ & $80.0 n(1)$ & 33,8 & 83,8 \\
\hline $\mathrm{LOF}(\mathrm{cm})$ & $0,7 \mathrm{~ns}$ & $3,9 *$ & 1 & 5,3 & 60,1 \\
\hline $\begin{array}{l}\text { LAF }(\mathrm{cm}) \\
\text { NEN } \\
\text { LOE }(\mathrm{cm}) \\
\text { HPL }(\mathrm{cm})\end{array}$ & $\begin{array}{l}7,6 * * \\
5,8 * * \\
4,6 *\end{array}$ & $\begin{array}{l}18,8^{* *} \\
7,9^{* *} \\
16,8^{* *} \\
4,6^{*}\end{array}$ & $\begin{array}{l}9,8 \\
7,7 \\
6,2\end{array}$ & $\begin{array}{l}21,2 \\
10,2 \\
19,4 \\
6,1\end{array}$ & $\begin{array}{l}68,6 \\
63,2 \\
71 \\
66,6\end{array}$ \\
\hline DTI (cm) & $\begin{array}{l}1,7 \mathrm{~ns} \\
4,4 *\end{array}$ & $4,9 *$ & $\begin{array}{l}2,5 \\
5,9\end{array}$ & 6,5 & 55,8 \\
\hline LOP (cm) & $2,4 \mathrm{~ns}$ & $12,6 * *$ & 3,4 & 15,3 & 78,3 \\
\hline $\begin{array}{l}\text { LAP }(\mathrm{cm}) \\
\text { PPA }(\mathrm{g})\end{array}$ & $1,6 \mathrm{~ns}$ & $\begin{array}{l}15,8 * * \\
3,7 *\end{array}$ & $\begin{array}{l}2,3 \\
47\end{array}$ & $\begin{array}{l}18,4 \\
5\end{array}$ & $\begin{array}{l}78,5 \\
63,7\end{array}$ \\
\hline $\begin{array}{l}\text { GPL }(\mathrm{g}) \\
\text { PCG }(\mathrm{g})\end{array}$ & $1,2 \mathrm{~ns}$ & $5 *$ & 1,7 & 6,6 & 62,3 \\
\hline
\end{tabular}

NJF : nombre de jours à $50 \%$ floraison, LOF : longueur de la $3^{\text {ieme }}$ feuille sous paniculaire, LAF : largeur de la $3^{\text {ième }}$ feuille sous paniculaire, NEN : nombre d'entre-nœuds, LOE : longueur de l'entre-nœud, HPL : hauteur de la plante, DTI : diamètre de la tige principale, LOP : longueur de la panicule , PPA : poids de la panicule principale, GPL : rendement en grains par plante, PCG : poids de cent grains, $\mathrm{O}$ cli : origine climatique des accessions, $\mathrm{F}$ : coefficient de Fischer, * : différence significative à 5\%, **: différence significative à 1\%, ns : différence non significative, $\mathrm{R}^{2}$ : coefficient de détermination. 
Tableau 6 : Corrélations entre les caractères quantitatifs de la collection de sorghos à grains sucrés du Burkina Faso.

\begin{tabular}{|c|c|c|c|c|c|c|c|c|c|c|c|}
\hline & NJF & LOF & LAF & NEN & LOE & HPL & DTI & LOP & LAP & PPA & GPL \\
\hline NJF & 1.00 & & & & & & & & & & \\
\hline $\mathrm{LOF}$ & 0.06 & 1.00 & & & & & & & & & \\
\hline LAF & $0.29 * *$ & $0.45 * *$ & 1.00 & & & & & & & & \\
\hline NEN & $0.44 * *$ & -0.03 & $0.48 * *$ & 1.00 & & & & & & & \\
\hline LOE & $-0.23 *$ & -0.13 & $-0.48^{* *}$ & $-0.35^{* *}$ & 1.00 & & & & & & \\
\hline HPL & -0.01 & -0.18 & -0.11 & $0.41 * *$ & $0.52 * *$ & 1.00 & & & & & \\
\hline DTI & 0.12 & $0.49 * *$ & $0.65 * *$ & $0.38 * *$ & $-0.41 * *$ & -0.01 & 1.00 & & & & \\
\hline LOP & $-0.24 *$ & 0.12 & $-0.29 * *$ & $-0.35 * *$ & $0.43 * *$ & $0.37 * *$ & -0.09 & 1.00 & & & \\
\hline LAP & -0.12 & $0.41 * *$ & $0.35 * *$ & 0.16 & $-0.21 *$ & $-0.24 *$ & $0.40 * *$ & $-0.27 * *$ & 1.00 & & \\
\hline PPA & $0.31 * *$ & $0.20 *$ & $0.43 * *$ & $0.39 * *$ & -0.13 & 0.19 & $0.24 *$ & -0.08 & 0.10 & 1.00 & \\
\hline GPL & $0.25 *$ & $0.25 *$ & $0.38 * *$ & $0.42 * *$ & -0.14 & $0.28 * *$ & $0.25 *$ & -0.04 & 0.04 & $0.76 * *$ & 1.00 \\
\hline PCG & $-0.60 * *$ & -0.08 & $-0.33 * *$ & $-0.32 * *$ & 0.19 & 0.04 & -0.16 & $0.23 *$ & -0.07 & $-0.33 * *$ & $-0.28 * *$ \\
\hline
\end{tabular}

*: Corrélation significatives au seuil de $5 \%$, **: corrélation significatives au seuil de $1 \%,, \mathrm{NJF}$ : nombre de jours à $50 \%$ floraison, LOF : longueur de la $3^{\text {ième }}$ feuille sous paniculaire, LAF : largeur de la $3^{\text {ième }}$ feuille sous paniculaire, NEN : nombre d'entre-nœuds, LOE : longueur de l'entrenœud, HPL : hauteur de la plante, DTI : diamètre de la tige principale, LOP : longueur de la panicule, LAP : largeur de la panicule principale, PPA : poids de la panicule principale, GPL : rendement en grains par plante, $\mathrm{PCG}$ : poids de cent grains.

Tableau 7: Performances moyennes des groupes de sorghos à grains sucrés du Burkina Faso.

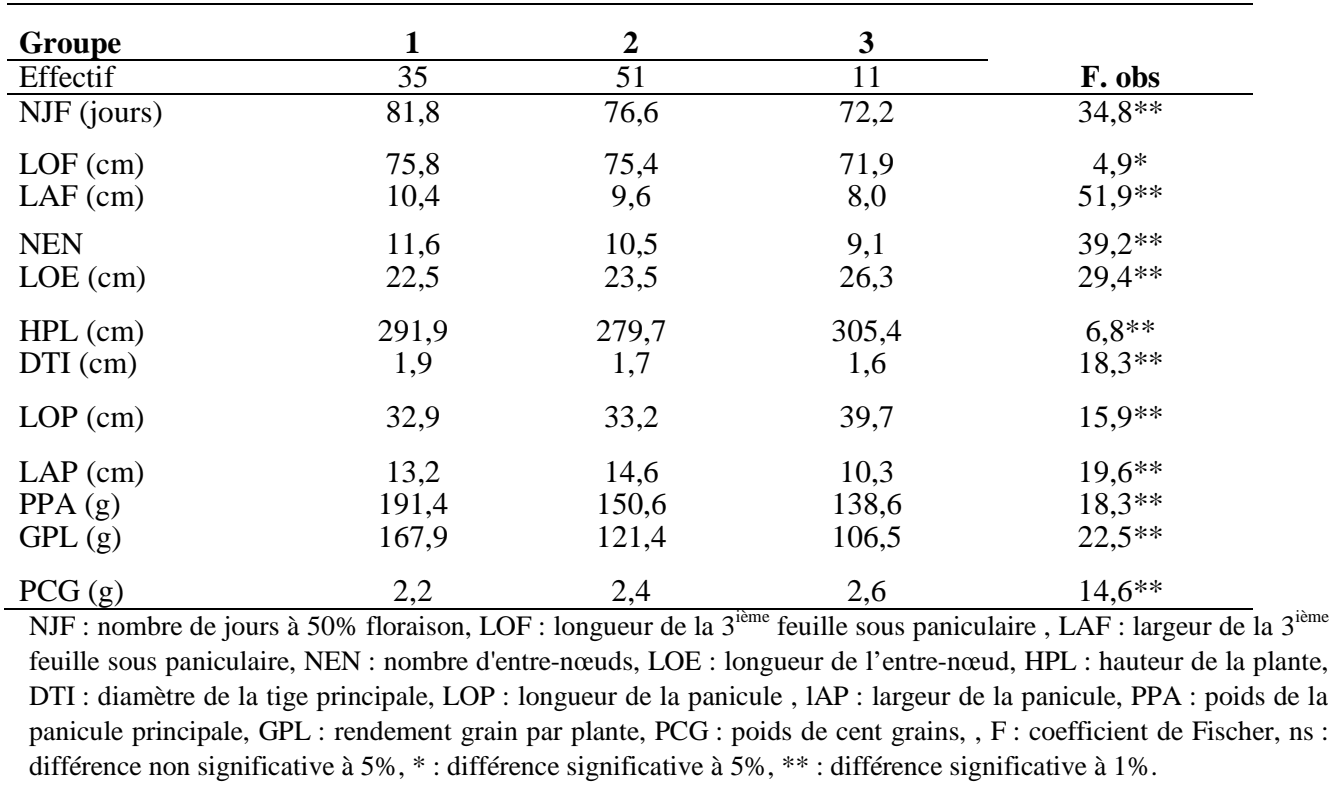


La faible superficie consacrée à la culture du sorgho à grains sucrés est liée à ses usages limités. En effet il est essentiellement un sorgho de bouche, récolté au stade grain pâteux pour être consommé frais. De ce fait, la monoculture est rare, l'association culturale est donc la plus fréquemment rencontrée. L'aspect farineux des grains de ce sorgho les rend très sensibles à l'attaque des ravageurs post-récolte notamment les insectes. Les paysans indépendamment des groupes ethniques ont donc développé plusieurs méthodes de conservation des semences pour limiter les dégâts. La conservation avec les panicules permet une meilleure protection des grains par les glumes et limite ainsi leur détérioration par les insectes. Ces mêmes observations sont rapportées par Nebié (2014) sur les sorghos à tige sucrée du Burkina Faso. La conservation des semences dans les enceintes hermétiques permet d'asphyxier les larves des ravageurs. Ces méthodes sont aussi signalées pour le voandzou (Touré et al., 2013).

\section{Diversité agromorphologique du sorgho à grains sucrés du Burkina Faso}

Un important polymorphisme est observé au niveau de huit caractères qualitatifs étudiés. Les caractéristiques morphologiques des panicules se rapprochent de celles du sorghum notabile définies par Snowden (1936). Les accessions appartiennent essentiellement à la race caudatum et à des hybrides inter races (caudatum-guinea, guinea-bicolor). Ces résultats diffèrent de ceux de Nebié et al. (2012) qui ont rapporté une fréquence élevée des races bicolor et des hybrides caudatumguinea au niveau des sorghos à grains sucrés du Centre Nord du Burkina Faso. Cela serait lié à la taille de son échantillon (27 écotypes). $\mathrm{La}$ forte proportion des intermédiaires (caudatum-guinea, guinea-bicolor) serait due au mode de gestion paysanne des accessions. En effet, les paysans produisent eux-mêmes leurs semences dans des champs où coexistent plusieurs cultivars. Cela favorise l'allogamie car malgré le mode de reproduction préférentiellement autogame, un important taux d'allofécondation variant de $7 \%$ à $40 \%$ est rapporté par Djé et al. (2004), Barnaud et al. (2007). Un échange de gènes s'effectue entre les accessions dans les champs des paysans du fait de l'association culturale.

Les différentes dénominations paysannes ne concordent pas avec les types botaniques définis par Harlan et De Wet (1972). Cela serait dû au fait que les critères utilisés dans la nomenclature paysanne ne correspondent pas à ceux de ces auteurs. Nos observations diffèrent cependant de celles de Teshome et al. (1997) en Ethiopie qui ont noté une concordance entre les différents noms vernaculaires locaux et les races principales de sorgho cultivé résultant d'une longue tradition ancestrale.

Le poids de la panicule principale et le rendement en grains par plante ont montré des coefficients de variation élevés. Tesso et al. (2011) ont également obtenu de forts coefficients de variation (> 30\%) pour ces mêmes caractères chez des sorghos de la race durra en Ethiopie. Ces résultats traduiraient une influence plus importante des facteurs environnementaux sur l'expression de ces caractères (Zongo, 1991). Les grains de ces sorghos ont un poids plus faible $(\mathrm{PCG}=2,4 \mathrm{~g})$ que ceux des sorghos non sucrés. Barro/Kondombo et al. (2008), Tesso et al. (2011) ont enregistré respectivement un poids de mille grains de $24.2 \mathrm{~g}$ pour les sorghos du centre ouest du Burkina Faso et 29.5 g pour des sorghos éthiopiens. Ces sorghos dont le cycle varie de 61 à 91 jours sont relativement plus précoces que les sorghos non sucrés dont le cycle varie de 58 à 135 jours (Zongo, 1991). Cette précocité du cycle fait des 
sorghos à grains sucrés, une culture intéressante pour les paysans pendant les périodes de soudure car elle arrive à maturité avant les autres sorghos et constitue une source importante de revenu monétaire pour les producteurs et les revendeurs.

La faible influence du facteur « origine climatique » dans l'expression de la variabilité des caractères serait en relation avec le mode de gestion des semences. En effet, dans les différentes zones de culture, ce sont principalement les paysans de l'ethnie mossi qui cultivent ces sorghos. Ils échangent non seulement les semences entre eux, mais en plus lors de leur exode vers d'autres zones, ils emportent avec eux leurs semences. Ils cultivent de ce fait des variétés semblables. La variation observée entre les accessions est donc essentiellement d'ordre génétique. Des résultats identiques ont été également obtenus par Nebié (2014) sur les sorghos à tige sucrée du Burkina Faso.

La corrélation positive observée entre le cycle et le rendement grains par plante pourrait s'expliquer par le cycle relativement court de ces sorghos qui a favorisé un très bon développement végétatif avec une très bonne activité photosynthétique et un bon remplissage des grains avant la fin de la saison des pluies. Cette hypothèse est confirmée par Quinby (1967) qui a montré qu'une bonne répartition des pluies dans le temps et dans l'espace occasionne de faibles rendements pour les sorghos de cycle court et de meilleurs rendements pour ceux de cycle long. Les physiologistes Cochème et Franquin (1967) ont également noté une évolution du cycle et du rendement dans le même sens en l'absence de tout facteur limitant. Des résultats similaires avaient été également obtenus par Tesso et al. (2011) sur des sorghos éthiopiens de la race durra. Au regard de cette corrélation et de la forte héritabilité du cycle, celui-ci pourrait être utilisé comme critère de sélection des accessions à haut rendement. Les corrélations positives observées entre les caractères peuvent faciliter l'amélioration génétique de tous les autres caractères corrélés positivement. Par contre, les corrélations négatives entrainent une diminution de la longueur de la panicule et du poids de cent grains avec le cycle. Ce qui pourrait limiter en sélection, les possibilités d'obtention de cultivars à cycle précoce et à haut rendement produisant des panicules longues et des grains lourds tant recherchés par les producteurs et surtout par les consommateurs.

\section{Organisation de la diversité agromorphologique des sorghos à grains sucrés}

La diversité agromorphologique observée entre les sorghos à grains sucrés du Burkina Faso a permis de les structurer en 3 groupes. Cette diversité est faible par rapport à celle des sorghos non sucrés et sorghos à tige sucrée du Burkina. Barro/Kondombo (2010) sur les sorghos non sucrés du centre ouest et Nebié (2014) sur les sorghos à tige sucrée du Burkina avaient distingué chacun 5 groupes morphologiques sur un échantillon respectif de 124 et 114 accessions. Les sorghos à grains sucrés sont donc moins diversifiés que les autres sorghos.

Les accessions du groupe III précoces fleurissent à partir de 72 jours après semis, les accessions du groupe II intermédiaires ont un cycle semis floraison de 77 jours et les accessions du groupe I plus tardives fleurissent à partir de 82 jours. Les accessions des groupes III sont les moins productives alors que celles du groupe I sont les plus productives. Les accessions du groupe II présentent les performances moyennes entre ces deux groupes. La sélection pourrait s'orienter vers les accessions du groupe I à haut rendement et du groupe III à cycle court 
pour une éventuelle hybridation afin d'avoir des cultivars à cycle court et à haut rendement grains.

\section{Conclusion}

Il ressort de notre étude que les paysans utilisent plusieurs techniques de conservation des semences et attribuent une diversité de noms vernaculaires aux sorghos à grains sucrés basés surtout sur des caractères phénotypiques visibles. Une corrélation intéressante entre le cycle et les paramètres du rendement et une importante diversité agromorphologique entre les accessions ont été également observées. Les accessions de la collection peuvent être reparties en 3 groupes agromorphologiques. Le groupe I présente le cycle le plus long et le meilleur rendement grains par plante alors que le groupe III est le plus précoce et le moins productif. Il a également été noté une faible influence de la diversité agromorphologique par le facteur origine climatique. Une évaluation à partir d'effectifs plus équilibrés des différentes zones agro-écologiques et des essais multilocaux permettront de mieux cerner l'effet des facteurs climatiques et de la race sur l'expression de ces caractères.

Enfin, l'identification et la caractérisation des sucres présents dans les grains, complétée par une caractérisation des accessions par des marqueurs moléculaires pourraient permettre de mieux approfondir la présente étude de diversité. Ces études complémentaires permettraient d'identifier les meilleures stratégies de sélection et d'orienter les sélectionneurs sur le choix de certains génotypes d'intérêt pour une gestion efficiente et une exploitation durable de leurs ressources génétiques dans les programmes d'amélioration du sorgho.

\section{REMERCIEMENTS}

Nous remercions sincèrement l'Institut du Développement Rural (IDR) pour nous avoir permis de réaliser l'expérimentation sur leur site à Gampèla. Nos remerciements vont également aux doctorants KIEBRE Zakaria, TIAMA Djakaridia, SAWADOGO Boureima de l'Equipe Génétique et Amélioration des Plantes du Laboratoire Biosciences de l'Université de Ouagadougou pour leurs suggestions et critiques dans la rédaction du manuscrit et KADEBA Abel du Laboratoire Botanique et Ecologie Végétales de l'Université de Ouagadougou pour son appui dans la réalisation de la carte des zones agroécologiques. Nous manifestons également notre reconnaissance aux techniciens YANOGHO Gilles et NIKIEMA Oumarou pour leur appui dans le suivi de l'essai et aux producteurs qui ont bien accepté partager leurs connaissances et donner leurs semences.

\section{RÉFÉRENCES}

Abu HB, Buah SSJ. 2011. Characterization of Bambara Groundnut Landraces and Their Evaluation by Farmers in the Upper West Region of Ghana. Journal of Developments in Sustainable Agriculture, 6: 64-74.

Aljane F, Ferchini A. 2007. Caractérisation et évaluation de six cultivars du caprifiguier (Ficus carica L.) en Tunisie. Plant Genetic Resource Newsletter, 151: 22-26.

Barnaud A, Deu M, Garine E, Mckey D, Joly HI. 2007. Local genetic diversity of sorghum in a village in northern Cameroon: structure and dynamics of landraces. Theoretical and Applied Genetics, 114: 237-248.

Barro/Kondombo CP. 2010. Diversité agromorphologique et génétique de variétés locales de sorgho (Sorghum bicolor [L.] Moench) au Burkina Faso. Eléments pour la valorisation des ressources génétiques 
locales. Thèse de doctorat, Université de Ouagadougou, p. 112.

Barro/Kondombo CP, Broke KV, Chantereau J, Sagnard F, Zongo JD. 2008. Variabilité phénotypique des sorghos locaux de deux régions du Burkina Faso: la Boucle du Mouhoun et le Centre-Ouest. Cahiers d'Agricultures, 17(2): 107-113.

Cocheme J, Franquin P. 1967. A study of the agroclimatology of semi-arid area South of the Sahara in West Africa. FAO/UNESCO, p. 325.

Deu M, Sagnard F, Chantereau J, Calatayud C, Hérault D, Mariac C, Pham JL, Vigouroux Y, Kapran I, Traoré PS, Mamadou A, Gérard B, Ndjeunga J, Bezançon G. 2008. Niger-wide assessment of in situ sorghum genetic diversity with microsatellite markers. Theor. Appl. Genet., 116: 903-916.

Djè Y, Heuertz $M$, Ater $M$, Lefèbvre $C$, Vekemans X. 2004. In situ estimation of outcrossing rate in sorghum landraces using microsatellite markers. Euphytica, 138: 205-212.

Djè Y, Heuertz M, Ater M, Lefebvre C, Vekemans X. 2007. Évaluation de la diversité morphologique des variétés traditionnelles de sorgho du Nord-ouest du Maroc. Biotechnol. Agron. Soc. Environ., 11(1): 39-46.

Faostat. 2012. http://faostat.fao.org visité en février 2012.

Frankel O, Brown AHD, Burdon JJ. 1995. The Conservation of Plant Biodiversity. Cambridge University Press: New York, USA; 299.

Harlan JR, De Wet JMJ. 1972. Une classification simplifiée des sorghos cultivés. Crop Science, 9(2): 172-176.

House LR. 1987. Manuel pour la Sélection du Sorgho ( $2^{\text {ème } e ́ d n) . ~ I C R I S A T: ~ P a t a n c h e r u ; ~}$ 229.
IBPGR/Icrisat. 1993. Descriptors for Sorghum [Sorghum bicolor (L) Moench]. International Board for Plant Genetic Ressources: Rome, Italy; International Crops Research Institute for the SemiArid Tropics: Patancheru, India; 38.

Jiro H, Sawadogo M, Millogo J, 2011. Caractérisations agromorphologique et anatomique du gombo du Yatenga et leur lien avec la nomenclature locale des variétés. Sciences \& Nature, 8(1): 23 36.

Missihoun AA, Agbangla C, AdoukonouSagbadja H, Ahanhanzo C, Vodouhe R, 2012. Gestion traditionnelle et statut des ressources génétiques du sorgho (Sorghum bicolor L. Moench) au NordOuest du Bénin. Int. J. Biol. Chem. Sci., 6(3): 1003-1018.

Nanema KR. 2010. Ressources génétiques de Solenostemon rotundifolius (Poir.) J. K. Morton du Burkina Faso: système de culture, variabilité agro-morphologique et relation phylogénétique entre les différents morphotypes cultivés au Burkina. Thèse Unique, Univ. Ouaga (Burkina Faso), p. 122.

Nebié B. 2014. Diversité génétique d'une collection de sorgho a tige sucrée [Sorghum bicolor (L.) Moench] du Burkina Faso. Thèse Unique, Université de Ouagadougou (Burkina Faso), p. 118.

Nebié B, Gapili N, Traoré RE, Nanema KR, Bationo/Kando P, Sawadogo M, Zongo JD. 2012. Diversité phénotypique des sorghos à grains sucrés du centre nord du Burkina Faso. Sciences et Techniques, Sciences Naturelles et Agronomie, 32(12): 2010-2012.

Nebié B, Nanema KR, Bationo/Kando P, Traoré RE, Labeyrie V, Sawadogo N, Sawadogo M, Zongo JD. 2013. Variation des caractères agromorphologiques et du brix d'une collection de sorghos à tige 
sucrée du Burkina Faso. Int. J. Biol. Chem. Sci., 7(5): 1919-1928.

Ouédraogo JT, Sawadogo M, Tignégré JB, Drabo I, Balma D. 2010. Caractérisation agro-morphologique et moléculaire de cultivars locaux de niébé (Vigna unguiculata) du Burkina Faso. Cameroon Journal of Experimental Biology, 6(01): 31-40.

Quinby JR. 1967. The maturity genes of sorghum. Advances in Agronomy, 19: 267-305.

Sawadogo M, Balma D. 2003. Etude agromorphologique de quelques écotypes locaux de gombo cultivés au Burkina Faso. Science et Technique, Série Sciences Naturelles et Agronomie, 27(12): 111-129.

Snowden JD. 1936. The Cultivated Races of Sorghum. Adlard: London, UK; 272.

Stanfield WD. 1983. Genetics. McGrawHillInc: New York; 281.

Teshome A, Baum BR, Fahrig L, Torrance JK, Arnason TJ, Lambert JD. 1997. Sorghum (Sorghum bicolor (L.) Moench) landrace variation and classification in north Shewa and south Welo, Ethiopia. Euphytica, 97: 255-263.

Tesso T, Tirfessa A, Mohammed H. 2011. Association between morphological traits and yield components in the durra sorghums in Ethiopia. Hereditas, 148: 98109.

Touré Y, Koné M, Silué S, Kouadio YJ. 2013. Prospection, collecte et caractérisation agromorphologique des morphotypes de voandzou (Vigna subterranea (L.) Verdc. (Fabaceae) de la zone salvanicole en Côte d'Ivoire. European Scientific Journal, 9(24): 308-325.

Traoré ER. 2014. Etude de la diversité du taro (Colocasia esculenta (L.) Schott.) : cas d'une collection du Burkina Faso et d'une collection internationale. Thèse Unique, Université de Ouagadougou (Burkina Faso), p. 128

Zongo JD. 1991. Ressources génétiques des sorghos [Sorghum bicolor (L.) Moench] $d u$ Burkina Faso: Evaluation agromorphologique et génétique. Thèse d'état, Université d'Abidjan, p. 175. 